\title{
Right-to-left shunt and the hypercoagulable state: does paradoxical embolism play a role in patients with antiphospholipid syndrome and stroke?
}

\author{
Shunt direita-esquerda e hipercoagulabilidade: embolia paradoxal pode ser responsável \\ pelo AVC na síndrome antifosfolipídica?
}

Laura Nicoleti Zamproni, Viviane Flumignan Zétola, Marcos Christiano Lange

\begin{abstract}
Objective: Patent foramen ovale is associated with paradoxical embolism (PE) and stroke. Hypercoagulable states, such as antiphospholipid syndrome (APS), can exacerbate PE by increasing clot formation. The aim of this study was to verify whether patients with APS and stroke present a right-to-left shunt (RLS) with greater frequency than patients with APS but without stroke. Methods: Fifty-three patients with APS were tested for RLS using contrast-enhanced transcranial Doppler (CTCD): 23 patients had a history of stroke (Stroke Group) and 30 had no history of stroke (No-stroke Group). Results: cTCD was positive in 15 patients (65\%) from the Stroke Group and in 16 patients (53\%) in the No-stroke Group ( $p=0.56)$. The proportion of patients with a small RLS ( $\leq 10$ high-intensity transient sign or HITS) and a large RLS (>10 HITS) was similar between the groups without significant difference. Conclusions: Our data do not support the theory that paradoxical embolism may play an important role in stroke in APS patients.
\end{abstract}

Key words: antiphospholipid syndrome, stroke, paradoxical embolism, right-to-left shunt.

\section{RESUMO}

Objetivo: O forame oval patente está associado com embolia paradoxal e acidente vascular cerebral isquêmico (AVCi). Estados de hipercoagulabilidade, como a síndrome antifosfolipídica (SAF), podem facilitar esse processo, aumentando a formação de coágulos. O objetivo deste estudo foi verificar se pacientes com SAF e AVCi apresentam maior frequência de shunt direita-esquerda (SDE), comparados a pacientes com SAF sem AVCi. Métodos: Cinquenta e três pacientes com SAF foram testados para SDE usando Doppler transcraniano contrastado (DTCc): 23 com AVCi (Grupo AVC) e 30 sem história de AVCi (Grupo Controle). Resultados: DTCc foi positivo em 15 pacientes (65\%) do Grupo AVC e em 16 pacientes (53\%) no Grupo Controle ( $p=0,56$ ). A proporção de pacientes com pequeno SDE ( $\leq 10$ HITS) e grande SDE (>10 HITS) foi semelhante nos dois grupos. Não houve diferença significativa entre os grupos. Conclusões: Nossos dados não sugerem que embolia paradoxal seja causa importante de AVCi em pacientes com SAF.

Palavras-Chave: síndrome antifosfolipídica, acidente vascular cerebral isquêmico, embolia paradoxal, shunt direita-esquerda.

Patients with cryptogenic arterial ischemic stroke have a high prevalence of a patent foramen ovale (PFO) ${ }^{1}$. However, the simple existence of a right-to-left shunt (RLS) does not appear sufficient to explain the increased risk of ischemic stroke, and other significant factors must be required ${ }^{2}$. A hypercoagulable state could be one of these factors, because it increases thrombogenic mechanisms, such as venous thrombosis, which is related to paradoxical embolism $(\mathrm{PE})^{3}$.

Antiphospholipid syndrome (APS) is one of the most frequently acquired thrombophilias ${ }^{4}$. The presence of antiphospholipid antibodies (aPL) is associated with ischemic stroke ${ }^{47}$. Nevertheless, the mechanism underlying stroke in these patients remains unclear, and there is a lack of data in the literature on the role that PE may play in the pathophysiology of arterial ischemic stroke in APS patients. Therefore, the association of stroke with the combined presence of APS and an RLS is of interest.

The aim of this study was to verify whether APS patients who have experienced stroke present with a higher frequency of RLS than patients with APS without a history of stroke.

Neurology Division, Hospital de Clínicas, Federal University of Paraná, Curitiba PR, Brazil.

Correspondence: Laura Nicoleti Zamproni; Hospital de Clínicas, Serviço de Neurologia; Rua General Carneiro 181 / 40 andar; 80060 -900 Curitiba PR - Brasil; E-mail: Inzamproni@yahoo.com.br

Conflict of interest: There is no conflict of interest to declare.

Received 28 August 2011; Received in final form 17 February 2012; Accepted 24 February 2012 


\section{METHODS}

This is a cross-sectional study conducted prospectively. All patients diagnosed with APS and followed at the outpatient clinic of the Neurology and Rheumatology Division of the Hospital de Clínicas, Federal University of Paraná, were recruited between March 2009 and December 2010. After receiving written, informed consent, patients were evaluated by a single neurologist from the same institution and examined for the presence of an RLS. The Local Ethics Committee approved this study.

An APS diagnosis was made when the subject fulfilled the modified Sapporo criteria ${ }^{8}$ (at least, one clinical criterion and one laboratory criterion must have been met). The laboratory criteria were assessed using the standards and benchmarks provided by the local laboratory that processed the patient samples. The anticardiolipin IgG or IgM value was considered positive when greater than 10 GPL or MPL. That value is adopted in our laboratory although the literature only considers values over $40 \mathrm{GPL}$ or MPL as positive. Several articles in the literature have discussed the lack of standardization of anticardiolipin tests, the difference in results between centers and especially the difference between the kits utilized ${ }^{9}$. This reference value was maintained in our laboratory, however all patients included who had anticardiolipin antibody values between 10 and $40 \mathrm{GPL}$ or MPL also had a positive lupus anticoagulant ratio and a clinical history that was highly suggestive of APS. The level of lupus anticoagulant was considered positive when its ratio was greater than one, provided that the patient was not receiving heparin. If the patient was receiving oral anticoagulants, levels of international normalized ratio (INR) of up to 3.0 were accepted as a positive lupus anticoagulant test. If the patient had an INR that was greater than 3.0, the positive lupus anticoagulant test was not accepted, the anticoagulation treatment was adjusted and the lupus anticoagulant test was repeated.

Clinical and neurological evaluations were conducted on all cases, and no patients indicated the presence of an extracranial or an intracranial stenosis in their ultrasonography results. All patients had a transthoracic echocardiogram, but no patient had any significant alteration. All patients with a positive stroke history were previously evaluated to exclude other etiologies before they were considered as having an arterial ischemic stroke due to APS and, thus, were included in the current study. All patients underwent an additional brain computed axial tomography (CT). Five patients in each group had a magnetic resonance image (MRI) in the last two years, and Flair and diffusion sequences of these exams were also analyzed. Ischemic stroke was considered to be present when the patient had a previous history of a sudden neurologic deficit accompanied by an arterial territory lesion on CT or MRI. Arterial territories were classified into three clusters for analysis: cortical, when the stroke included a distal lesion; subcortical for lesions greater than 1,5 mm and not involving the cortex; and microvessels, when the lesions were smaller than $1,5 \mathrm{~mm}$. The patients were classified into two groups based on stroke investigation: Stroke Group, composed of patients with APS with a confirmed history of stroke; and Nostroke Group, composed of patients with APS with normal brain images and without a history of stroke.

The RLS investigation was performed by a single neurologist (it was not involved patients clinical evaluation) with expertise in contrast-enhanced transcranial Doppler (cTCD) ultrasonography. The cTCD (DWL Doppler-Box, Singen, Germany) procedures were performed while the patients were in a supine position, and the neurologist was blinded to the patients' medical status. Two 2-MHz-pulsed Doppler transducers were fixed using a head frame (DWL DiaMon, Singen, Germany), and the main stems of both middle cerebral arteries (MCAs) were insonated through the temporal window at a depth of 55 to $65 \mathrm{~mm}$ to capture a small sample volume of $12 \mathrm{~mm}$ in length with M-mode.

The contrast agent (CA) was composed of a mixture of $9 \mathrm{~mL}$ saline and $1 \mathrm{~mL}$ air. Prior to the infusion, the solution was prepared by agitating the mixture 10 times between two 10-mL syringes via a three-way tap, which was connected to a 24-gauge intravenous catheter that was inserted into the superficial vein of the patient's arm. The distance from the catheter to the syringes was less than $10 \mathrm{~cm}$. The $\mathrm{CA}$ was injected over five seconds into the antecubital vein.

The procedure was conducted while the patient was resting (resting phase) and before he performed a Valsalva maneuver (VM) protocol. The VM was performed five seconds after the CA was injected, and its effectiveness was confirmed by a $25 \%$ decrease of MCA flow velocity and by a deflection of at least $40 \mathrm{mmHg}$ in a manometer that the patient exhaled into during the VM. Both studies (resting phase and VM phase) were repeated three times, with each test lasting one minute.

Patients were considered RLS-positive when at least one high-intensity transient sign (HITS) was detected on the spectral display of at least one of the monitored MCAs. Conversely, the patient was determined to have no RLS (RLS negative) when, during the 60 seconds following the injection of the CA, there was no identified HITS in either MCA. Based on the cTCD results, the patients were classified as RLS-negative, "small RLS" when the patient presented with an RLS that was identified to be small ( $\leq 10$ bubbles) or "large RLS" when the patient's RLS was identified to be large ( $>10$ bubbles).

Exclusion criteria comprised the following items: no temporal window; a lack of peripheral venous access; or patients were restricted to the bed or with serious sequelae that were incompatible with the examination; or self-exclusion.

Statistical analyses were performed using the Statistica 6.0.software. Statistical significance was assessed using chisquared tests, Student's t-tests or Mann-Whitney tests. Statistical significance was determined using a critical value of $\mathrm{p}<0.05$. 


\section{RESULTS}

During the study period, 53 patients fulfilled the criteria for APS. No patient was excluded. Of these APS patients, 23 had a history of stroke (Stroke Group), and 30 had no history of stroke (No-stroke Group). In the Stroke Group, there were 20 women, and the mean \pm standard deviation (SD) age was $42 \pm 12$ years old. Six patients had APS secondary to systemic lupus erythematosus (SLE), and 17 patients had primary APS. Neuroimaging results revealed that only $40 \%$ of patients had multiple ischemic lesions, and $30 \%$ had lesions of different arterial territories (with $75 \%$ of the cases indicating a stroke in the anterior circulation). The stroke was cortical in 53\%, subcortical in $30 \%$ and in the basal ganglia in $17 \%$ of patients. In addition to stroke, another thrombotic event was found in $13 \%$ of patients, and gestational morbidity was found in $17 \%$ of patients.

In the No-stroke Group, there were 22 women, and the mean \pm SD age was $38 \pm 11$ years. Fourteen patients had APS secondary to SLE, one patient had APS secondary to rheumatoid arthritis, and 15 patients had primary APS. The main clinical event present in 20 patients was deep venous thrombosis (DVT). Four patients presented with a pulmonary embolism, three patients exhibited a cerebral venous thrombosis (without brain venous ischemia), and eight presented with gestational morbidity. There was no significant difference between Stroke Group and No-stroke Group. These demographics are presented in Table 1.

cTCD was positive in 15 (65\%) patients from the Stroke Group and in 16 (53\%) from the No-stroke Group ( $\mathrm{p}=0,56)$. In the Stroke Group, 8 patients showed the presence of an RLS confirmed by a resting test, and all 15 patients had confirmed RLS using the VM test. When separated into different grades of RLS, this group was comprised of eight patients with a small RLS and seven with a large RLS. In the No-stroke Group, small RLS and large RLS were observed in eight patients each. There was no significant difference between the No-stroke and Stroke Groups. These results are shown in Table 2.

In the Stroke Group, the patients with only one ischemic territory $(n=14)$ had a small RLS in five cases and a large RLS in four cases; five patients were RLS-negative. In patients with multiple ischemic territories $(n=9)$, three patients had a small RLS, three had a large RLS, and the other three patients were RLS-negative, as shown in Table 3.

Eighteen patients had an anterior circulation stroke: nine patients had cortical lesions, six patients had subcortical lesions, and three patients had microvascular lesions. Approximately $50 \%$ of patients with a cortical and subcortical anterior circulation stroke and $100 \%$ of basal ganglia stroke cases were RLS-positive. In the five patients with a posterior circulation stroke, three had cortical lesions, and two exhibited brainstem (BS) lesions. We found that $66 \%$ of patients with a cortical posterior circulation stroke and
Table 1. Demographics of APS patients, separated into Stroke and No-stroke Groups.

\begin{tabular}{lccc} 
& $\begin{array}{c}\text { Stroke } \\
\text { Group }\end{array}$ & $\begin{array}{c}\text { No-stroke } \\
\text { Group }\end{array}$ & p-value \\
\hline Patients & 23 & 30 & - \\
Female & 20 & 22 & 0.38 \\
Age (y) & $42 \pm 12$ & $38 \pm 11$ & 0.29 \\
Primary APS & 17 & 15 & 0.14 \\
Secondary APS & 6 & 15 & 0.14 \\
SLE & 6 & 14 & 0.21 \\
Duration of APS (y) & $5 \pm 6$ & $5 \pm 5$ & 0.75 \\
\hline Gestational morbidity & $4(17 \%)$ & $8(26 \%)$ & 0.64 \\
\hline DVT history & $2(8 \%)$ & $20(66 \%)$ & 0.0001 \\
\hline Time since stroke $(y)$ & $8 \pm 7$ & - & -
\end{tabular}

y: years; APS: antiphospholipid syndrome; SLE: systemic lupus erythematosus; DVT: deep venous thrombosis.

Table 2. RLS in Stroke and No-stroke Groups.

\begin{tabular}{lccc} 
& $\begin{array}{c}\text { Stroke } \\
\text { Group }\end{array}$ & $\begin{array}{c}\text { No-stroke } \\
\text { Group }\end{array}$ & p-value \\
\hline Patients & 23 & 30 & \\
RLS-positive & 15 & 16 & 0.56 \\
Positive in resting test & 8 & 10 & 0.85 \\
Only positive in VM test & 7 & 6 & 0.58 \\
Small RLS & 8 & 8 & 0.74 \\
Large RLS & 7 & 8 & 0.99 \\
\hline
\end{tabular}

RLS: right-to-left shunt; VM: Valsalva maneuver.

Table 3. RLS in Stroke Group.

\begin{tabular}{lccc} 
& $\begin{array}{c}\text { Single } \\
\text { lesion }\end{array}$ & $\begin{array}{c}\text { Multiple } \\
\text { lesions }\end{array}$ & p-value \\
\hline Patients & 14 & 9 & \\
RLS-negative in VM test & 5 & 3 & 0.63 \\
Small RLS in VM test & 5 & 3 & \\
Large RLS in VM test & 4 & 3 &
\end{tabular}

RLS: right-to-left shunt; VM: Valsalva maneuver.

Table 4. RLS and stroke characteristics in patients with APS.

\begin{tabular}{lcccc} 
Stroke territory & Patients & $\begin{array}{c}\text { RLS- } \\
\text { negative }\end{array}$ & $\begin{array}{c}\text { RLS- } \\
\text { positive }\end{array}$ & p-value \\
\hline Anterior circulation & 18 & & & \\
Cortical & 9 & $4(45 \%)$ & $5(55 \%)$ & 0.5 \\
Subcortical & 6 & $3(50 \%)$ & $3(50 \%)$ & \\
Microvascular & 3 & $0(0 \%)$ & $3(100 \%)$ & \\
Posterior circulation & 5 & & & \\
Cortical & 3 & $1(33 \%)$ & $2(66 \%)$ & 0.7 \\
BS & 2 & $1(50 \%)$ & $1(50 \%)$ & \\
\hline
\end{tabular}

RLS: right-to-left shunt; APS: antiphospholipid syndrome; BS: brainstem. 
$50 \%$ of patients with a BS stroke were RLS-positive. No difference was noted between the presence of an RLS and the pattern of ischemic stroke (Table 4).

\section{DISCUSSION}

In this study, there was no relationship between stroke and an RLS in patients with APS, even when multiple ischemic territories or different lesion patterns were present.

PFO can be associated with cryptogenic stroke; the mechanism is postulated to be a $\mathrm{PE}$, where clots originate in the venous circulation and travel to the arterial side through an RLS ${ }^{10,11}$. Systemic hypercoagulable states can potentially increase clot formation $^{12}$. It is reasonable to hypothesize that patients with hypercoagulable states may be at a higher risk of developing a paradoxical embolism and stroke. To our knowledge, there is no literature to prove this association ${ }^{13,14}$. Most of the published data on this issue are either anecdotal or case series ${ }^{12}$.

Di Tullio et al. ${ }^{15}$ reported a significantly higher prevalence of protein $\mathrm{C}$ deficiency in 25 stroke patients with PFO compared with 195 without PFO. However, this study diagnosed the presence of RLS using transthoracic contrast echocardiography, which may introduce substantial bias. Barinagarrementeria et al. ${ }^{16}$ and Chaturvedi ${ }^{11}$ obtained similar findings in a small case series, whereas Schwarze et al. ${ }^{17}$ found an increased prevalence of RLS and activated protein C resistance in patients with stroke compared with patients without this thrombophilic disorder.

Florez et al. ${ }^{13}$ studied patients with stroke and verified the coexistence of PFO and a hypercoagulable state. The frequency of a hypercoagulation abnormality was not higher in patients with a PFO compared with patients without a PFO. Similar results were obtained by Carod-Artal et al. ${ }^{18}$. In their study, the prevalence of thrombophilia in PFOpositive and PFO-negative patients with cryptogenic stroke and stroke patients with known etiology was examined. The authors found no difference between the PFO-positive and PFO-negative groups ${ }^{18}$. Notably, a group of control subjects with no history of stroke was lacking in all of these studies.
Recently, the PICCS-APASS study analyzed the presence of APL antibodies and a PFO in stroke patients. This study did not report a relationship among positive APL antibodies, PFO and the recurrence of cerebral ischemic events ${ }^{14}$. Notwithstanding, this study considered the only presence of APL antibodies and did not consider patients with diagnosed APS.

In our study, patients with confirmed APS, with and without a history of stroke, were evaluated for the presence of an RLS. We also attempted to correlate the characteristics of arterial stroke with the presence of an RLS. The data did not support the theory that patients with APS who had experienced stroke have a greater frequency of RLS than patients with APS who did not have a history of cerebral ischemic events. No relationship was found between multiple ischemic events and cortical stroke and the presence of an RLS. Interestingly, a high prevalence of RLS was found in both groups with APS (65 and 53\%) versus the population prevalence estimated in the literature $(30 \%)^{12}$. Unfortunately, there is no data in the literature comparing the prevalence of RLS in the APS population versus healthy subjects. It is well known that APS predisposes patients to cardiac defects, such as valvar abnormalities. Although speculative, it is likely that APS patients also present with more cardiac shunts.

The limitations of this study include the small number of patients (APS is a rare disease) and the lack of transesophageal echocardiography to confirm the presence of a PFO. ß2-Glycoprotein testing was unavailable for patients in this study, because it is not routine in our institution.

The results of the current study do not support the hypothesis that paradoxical embolism plays an important role in stroke pathophysiology of APS patients. Other mechanisms, such as microvascular arterial thrombosis and cardiopathy, could underlie the stroke in these patients. More studies, with larger numbers of patients, are required to confirm these findings. However, these data suggest that the clinical treatment of patients with hypercoagulable disorders may play a more important role in the prevention of stroke than in the correction of RLS.

\section{References}

1. Cabanes L, Mas JL, Cohen A, et al. Atrial septal aneurysm and patent foramen ovale as risk factors for cryptogenic stroke in patients less than 55 years of age: a study using transesophageal echocardiography. Stroke 1993;24:1865-1873.

2. Ozdemir AO, Tamayo A, Munoz C, Dias B, Spence JD. Cryptogenic stroke and patent foramen ovale: clinical clues to paradoxical embolism. J Neurol Sci 2008;275:121-127.

3. The Antiphospholipid Antibodies in Stroke (APASS) Study Group. Anticardiolipin antibodies are an independent risk factor for first ischemic stroke. Neurology 1993;43:2069-2073.

4. Ruiz-Irastorza G, Khamashta MA. Stroke and antiphospholipid syndrome: the treatment debate. Rheumatology 2005;44:971-974.
5. Tuhrim S, Rand JH, Wu X, et al. Elevated anticardiolipin antibody titer is a stroke risk factor in a multiethnic population independent of isotype or degree of positivity. Stroke 1999;30:1561-1565.

6. Brey RL, Stallworth CL, McGlasson DL, et al. Antiphospholipid antibodies and stroke in young women. Stroke 2002;33:2396-2400.

7. Brey RL, Abbott RD, Curb JD, et al. Beta 2-glycoprotein 1-dependent anticardiolipin antibodies and risk of ischemic stroke and myocardial infarction: the honolulu heart program. Stroke 2001;32:1701-1706.

8. Miyakis S, Lockshin D,AtsumiT,etal. International consensus statement on an update of the classification criteria for definite antiphospholipid syndrome (APS). J Thromb Haemostasis 2006;4:295-306.

9. Pengo V, Biasiolo A, Bison E, et al. Antiphospholipid antibody ELISAs: 
survey on the performance of clinical laboratories assessed by using lyophilized affinity-purified IgG with anticardiolipin and anti-beta2Glycoprotein I activity. Thromb Res 2007;120:127-133.

10. Karttunen V, Hiltunen L, Rasi V, Vahtera E, Hillbom M. Factor V Leiden and prothrombin gene mutation may predispose to paradoxical embolism in subjects with patent foramen ovale. Blood Coagul Fibrinolysis 2003;14:261-268.

11. Chaturvedi S. Coagulation abnormalities in adults with cryptogenic stroke and patent foramen ovale. J Neurol Sci 1998;160:158-160.

12. Lange MC, Zamproni LN, Braatz V, Fernandes AF, Zetola VF, Nóvak EM. Ischemic stroke in a patient with Crohn's disease: a confirmed paradoxical embolism mechanism. Arq Neuropsiquiatr 2010;68:651-652.

13. Florez JC, Ay H, Van Cott EM, Buonanno FS. Patent foramen ovale and hypercoagulability as combined risk factors for stroke. J Stroke Cerebrovasc Dis 2003;12:114-118.
14. Rajamani K, Chaturvedi S, Jin Z, et al. Patent foramen ovale, cardiac valve thickening, and antiphospholipid antibodies as risk factors for subsequent vascular events: the PICSS-APASS study. Stroke 2009;40;2337-2342.

15. Di Tullio MR, Santoni-Rugiu F, Sacco RL, et al. Patent foramen ovale and hypercoagulable states in ischemic stroke patients. Circulation 1994;90:398.

16. Barinagarrementeria F, Santos J, Ruiz-Sandoval JL, et al. Prothrombotic states as triggering of cerebral infarction in patients with patent foramen ovale. Neurology 1998;50:155.

17. SchwarzeJJ, KlingelhoferJ, Kim S, et al. Coexistence of a prothrombotic state and right-to-left shunts: a potential mechanism of paradoxical embolism? Neurology 1998;50:A154.

18. Carod-Artal FJ, Villela Nunes S, Portugal D. Thrombophilia and patent foramen ovale in young stroke patients. Neurologia 2006;21:710-716. 